\title{
Effect of a High-Carbohydrate, Low-Saturated-Fat Diet on Apolipoprotein B and Triglyceride Metabolism in Pima Indians
}

\author{
William G. H. Abbott, Boyd Swinburn, Giacomo Ruotolo, Hitoshi Hara, Lidia Patti, \\ Ingeborg Harper, Scott M. Grundy, and Barbara V. Howard \\ Clinical Diabetes and Nutrition Section, National Institute of Diabetes, Digestive and Kidney Diseases, \\ National Institutes of Health, Phoenix, Arizona, 85016
}

\begin{abstract}
The mechanisms by which high-carbohydrate, low-saturatedfat diets lower LDL cholesterol (LDLC) concentrations are unknown. In this study, kinetics of VLDL, intermediate density lipoprotein (IDL), and LDL apoprotein B and VLDL triglyceride were determined in seven nondiabetic (ND) and seven non-insulin-dependent diabetic (NIDDM) Pima Indian subjects on high-fat and high-carbohydrate (HICHO) diets. Metabolic changes were similar in ND and NIDDM. On the HICHO diet, LDLC decreased $(131 \pm 8$ vs. $110 \pm 7 \mathrm{mg} / \mathrm{dl}, P$ $<0.0001)$ in all subjects. Mean fasting and 24-h triglyceride (TG) concentrations were unchanged, as were mean production rates and fractional clearance rates (FCR) of VLDL apoB and VLDL TG. The mean VLDL apoB pool size $(303 \pm 20$ vs. $371 \pm 38 \mathrm{mg}, P=0.01$ ) increased owing to a decrease in the mean transport rate $(10.7 \pm 1.1 \mathrm{vs.} 8.4 \pm 0.9 \mathrm{mg} / \mathrm{kg}$ fat-free mass (ffm) per day, $P<0.0001)$ and the mean rate constant $(2.3 \pm 0.2$ vs. 1.5 $\pm 0.2, P<0.001)$ for the VLDL apoB to IDL apoB conversion pathway. The mean transport rate of VLDL apoB to $L D L$ apoB via IDL $(10.2 \pm 0.9 \mathrm{vs} .8 .0 \pm 0.8 \mathrm{mg} / \mathrm{kg} \mathrm{fm}$ per day, $P<0.001)$ decreased. Mean LDL apoB concentrations decreased $(70 \pm 5$ vs. $61 \pm 5 \mathrm{mg} / \mathrm{dl}, P<0.001)$ on the HICHO diet. Means for total LDL apoB transport rate, LDL apoB FCR, and LDLC/apoB ratios were unchanged. In summary, the HICHO diet decreased the activity of mechanisms that convert VLDL to LDL, which contributed to the decrease in LDLC in all subjects. There was also evidence in some subjects for increased activity of LDL apoB clearance mechanisms, and a decrease in the LDLC to apoB ratio. (J. Clin. Invest. 1990. 86:642-650.) Key words: diabetes $\bullet$ dietary fat $\bullet$ lipoproteins
\end{abstract}

\section{Introduction}

When saturated fatty acids are removed from the diet and replaced by either polyunsaturated fatty acids (1-5), monounsaturated fatty acids $(5,6)$ or carbohydrates $(7,8)$, concentra-

Dr. Abbott's and Dr. Grundy's current address is Center for Human Nutrition, Southwestern Medical School, 5323 Harry Hines Boulevard, Dallas, TX 75239; Dr. Ruotolo's is Department of Medicine, Istituto Scientifico S. Raffaelle, Milan University School of Medicine, Via Olgettina 60, 20132, Milan, Italy; Dr. Howard's is Medlantic Research Foundation, 108 Irving St., N.W., Washington, DC 20010; Dr. Hara's is Second Department of Medicine, Hiroshima University School of Medicine, 1-2-3 Kasumi, Hiroshima, Japan 734; Dr. Patti's is Isitituto di Medicina e Malattie Dismetaboliche, Facoltà di Medicina e Chirurgia, Università di Napoli, Nuovo Policlinico, Via Sergio Pansini 5, 80131, Naples, Italy.

The Journal of Clinical Investigation, Inc.

Volume 86, August 1990, 642-650 tions of LDL cholesterol fall. The mechanisms where by the exchange of saturated fatty acids for other nutrients produce a decrease in LDL cholesterol concentrations are not well understood. The metabolic changes associated with the exchange of polyunsaturated for saturated fatty acids have been studied extensively (2-5). In recent years the substitution of carbohydrates for saturated fatty acids has been recommended for the purpose of LDL cholesterol lowering $(9,10)$. The metabolic consequences of this exchange have been studied less thoroughly.

The main purpose of this study was to determine the mechanisms whereby replacement of saturated fatty acids by complex carbohydrates produces a reduction in LDL cholesterol concentrations. We also wished to determine whether solid food, isocaloric, high carbohydrate diets cause an increase in either VLDL concentrations or hepatic VLDL production. This issue is of importance because high plasma triglyceride concentrations have been associated with increased atherosclerosis in diabetic patients $(11,12)$. The study was carried out in two groups of patients: those with non-insulin-dependent diabetes mellitus (NIDDM) ${ }^{1}$ and those without diabetes.

\section{Methods}

\section{Subjects}

The subjects for this study were 14 Southwestern American Indians from the Gila River Indian Reservation. All subjects had a full medical history and physical examination on admission to the study, with routine measurements in hematology and biochemistry, and urinalysis. Seven subjects had NIDDM diagnosed by oral glucose tolerance test by World Health Organization criteria (13). All subjects were free of significant disease apart from NIDDM, and none had significant complications of NIDDM. Oral hypoglycemic agents and insulin therapy were stopped $1 \mathrm{wk}$ before admission to the study. No subjects were taking drugs known to affect carbohydrate or lipid metabolism. The subjects' age, sex, weight, body composition, and fasting plasma glucose concentration just before the high-fat diet turnover study are shown in Table I.

\section{Experimental design}

Protocol. Each subject had two admissions to the research ward, at least $4 \mathrm{wk}$ apart. On one admission a high-fat diet was given for $5 \mathrm{wk}$, and on the other admission a low-fat, high-carbohydrate diet was given for between 5 and $7 \mathrm{wk}$. The high-carbohydrate diet was fed for a longer baseline period to allow plasma triglyceride concentrations to reach stable concentrations. Five subjects ate the high-fat diet first, and nine subjects ate the high-carbohydrate diet first. During the week after the baseline period ( 2 wk on the high-fat diet, and 2-4 wk on the high-carbohydrate diet) the subjects had a measurement of body composition,

1. Abbreviations used in this paper: FCR, fractional catabolic rate; ffm, fat-free mass; NIDDM, non-insulin-dependent diabetes mellitus. 


\begin{tabular}{|c|c|c|c|c|c|c|c|}
\hline \multirow[b]{2}{*}{ Subject } & \multirow[b]{2}{*}{ Age } & \multirow[b]{2}{*}{ Sex } & \multicolumn{2}{|c|}{ Weight } & \multicolumn{2}{|c|}{ Percent body fat } & \multirow{2}{*}{$\begin{array}{l}\text { Fasting } \\
\text { glucose }\end{array}$} \\
\hline & & & HIFAT & нІСно & HIFAT & НICHO & \\
\hline & $y r$ & & & & & & $m g / d l$ \\
\hline \multicolumn{8}{|l|}{ Nondiabetic } \\
\hline 1 & 28 & $\mathbf{F}$ & 110 & 110 & 39 & 41 & 88 \\
\hline 2 & 52 & $\mathbf{M}$ & 123 & 122 & 34 & 34 & 107 \\
\hline 3 & 24 & $\mathbf{M}$ & 89 & 89 & 26 & 25 & 95 \\
\hline 4 & 32 & $\mathbf{M}$ & 93 & 94 & 21 & 24 & 91 \\
\hline 5 & 31 & $\mathbf{M}$ & 131 & 135 & 35 & 38 & 101 \\
\hline 6 & 23 & $\mathbf{M}$ & 156 & 153 & 37 & 34 & 84 \\
\hline 7 & 44 & $\mathbf{M}$ & 117 & 114 & 35 & 31 & 101 \\
\hline Mean \pm SEM & $32 \pm 4$ & & $117 \pm 9$ & $117 \pm 8$ & $32 \pm 2$ & $32 \pm 2$ & $95 \pm 3$ \\
\hline \multicolumn{8}{|l|}{ NIDDM } \\
\hline 8 & 23 & $\mathbf{M}$ & 135 & 133 & 36 & 36 & 110 \\
\hline 9 & 49 & $\mathbf{F}$ & 81 & 80 & 48 & 46 & 102 \\
\hline 10 & 47 & $\mathbf{M}$ & 90 & 91 & 26 & 25 & 233 \\
\hline 11 & 44 & $\mathbf{F}$ & 89 & 88 & 43 & 41 & 340 \\
\hline 12 & 22 & $\mathbf{M}$ & 79 & 79 & 30 & 30 & 197 \\
\hline 13 & 42 & $\mathbf{M}$ & 71 & 71 & 22 & 21 & 285 \\
\hline 14 & 43 & $\mathbf{F}$ & 65 & 65 & 32 & 34 & 229 \\
\hline Mean \pm SEM & $39 \pm 4$ & & $87 \pm 9$ & $87 \pm 8$ & $34 \pm 4$ & $33 \pm 3$ & $214 \pm 33$ \\
\hline
\end{tabular}

Abbreviations: HICHO, high-carbohydrate diet; HIFAT, high-fat diet.

an oral glucose tolerance test, three measurements of fasting plasma lipoprotein concentrations, a 24 -h profile of triglyceride and glucose concentrations, and a 24-h urinary glucose estimation. After these tests, the kinetics of apolipoprotein B-100 (apoB) in VLDL, intermediate density lipoprotein (IDL), and LDL and of VLDL-triglyceride were measured.

Diets. Weight maintenance energy requirements were calculated from body weight. The fasting, posturination body weight was measured each morning, and these measurements were used to adjust caloric intake to maintain constant body weight. Diet composition is shown in Table II. Saturated fatty acids were derived predominantly from dairy products and were replaced with predominantly complex carbohydrate of vegetable, legume, and cereal origin, resulting also in an increase in fiber. Cholesterol content was the same on both diets. Meals were made from readily available foods, and diet composition was calculated from tables $(14,15)$.

\section{Procedures}

Body composition. Body composition was estimated by underwater weighing as previously described (16). This measurement was used to calculate each subject's fat-free mass (ffm), for normalization of kinetic data.

Oral glucose tolerance test. An oral glucose tolerance test was performed using $75 \mathrm{~g}$ of glucose (Koladex, Custom Laboratories, Maryland). Plasma glucose and insulin concentrations were measured at -5 and $120 \mathrm{~min}(16)$.

24- $h$ glucose and triglyceride profile. After a 12-h overnight fast, a catheter was inserted in an arm vein and kept patent with a slow saline infusion. Blood was drawn for measurement of plasma glucose and triglyceride concentrations at $\mathbf{0 7 1 5}$ hours, 0800 hours, and two hourly thereafter until 0600 hours the next morning. Meals were eaten at $0730,1130,1630$, and 1930 hours, with calories divided in the ratio 20:30:30:20.

Fasting plasma and lipoprotein lipid concentrations. Fasting plasma lipoproteins were separated by ultracentrifugation as previously described (16).
$V L D L$ and $L D L$ metabolic studies. VLDL apoB, IDL apoB, LDL apoB, and VLDL triglyceride kinetics were measured by a previously described method (17). At the end of the baseline period, $500 \mathrm{ml}$ of venous blood was collected into citrate dextrose. Red blood cells were reinfused. VLDL $(d<1.006)$ and LDL $(d 1.019-1.063)$ were isolated from the plasma by preparative ultracentrifugation. VLDL were labeled with ${ }^{131} I$ and LDL with ${ }^{125} I$ (18). The iodinated lipoproteins were

Table II. Diets

\begin{tabular}{lrr} 
& \multicolumn{2}{c}{ Diet } \\
\cline { 2 - 3 } Component & High fat & High carbohydrate \\
\hline Carbohydrate $(\%)$ & 43 & 65 \\
Total $(g)$ & 322 & 498 \\
Simple $(g)$ & 102 & 148 \\
Complex $(g)$ & 220 & 350 \\
Fat $(\%)$ & 42 & 21 \\
Polyunsaturated $(\%)$ & 6 & 6 \\
Monounsaturated $(\%)$ & 12 & 8 \\
Saturated $(\%)$ & 21 & 6 \\
Protein $(\%)$ & 15 & 15 \\
Animal/vegetable $(\%)$ & $58: 42$ & $41: 59$ \\
Fiber & & 23 \\
Cereal $(g)$ & 8 & 22 \\
Vegetable $(g)$ & 12 & 52 \\
Legume $(g)$ & 9 & 23 \\
Cholesterol $(m g)$ & 560 & \\
& & \\
\hline
\end{tabular}

Weights are for 3,000-calorie diet.

High-Carbohydrate Diet and Apolipoprotein B Metabolism 
diluted in the patient's own serum, filtered, and tested for sterility and pyrogenicity.

$18 \mathrm{~h}$ before the VLDL injection, a fat-free, liquid formula diet was started that provided the same number of carbohydrate calories and the same number of protein calories as the subject ate on the weightmaintenance solid-food diet. The formula feedings started at 1500 hours and were given in 14 three-hourly feedings over the next $42 \mathrm{~h}$. The solid-food, weight-maintenance diet was then restarted. $18 \mathrm{~h}$ after starting the formula diet (0900 hours), $25 \mu \mathrm{Ci}$ of ${ }^{131} \mathrm{I}-\mathrm{VLDL}$ and 300 $\mu \mathrm{Ci}$ of tritiated glycerol were injected. $25 \mu \mathrm{Ci}$ of ${ }^{125} \mathrm{I}-\mathrm{LDL}$ was injected $48 \mathrm{~h}$ later. The specific activities of ${ }^{131} \mathrm{I}$-VLDL apoprotein $\mathrm{B}$ and VLDL triglyceride were measured in 16 samples taken during the $48 \mathrm{~h}$ after the VLDL injections. The specific activity of ${ }^{131}$ I-IDL $(d 1.006-$ 1.019) apoprotein $B$ was measured in 24 plasma samples taken during the $4 \mathrm{~d}$ after the VLDL injections. Peak counts of ${ }^{131} \mathrm{I}$-IDL were between 500 and $1,000 \mathrm{cpm}$. Once three consecutive points were $<50 \%$ above background all further points were excluded. The specific activity of ${ }^{131} \mathrm{I}$-LDL apoprotein B was measured in all plasma samples taken during the $16 \mathrm{~d}$ of the study. The peak counts for ${ }^{131} \mathrm{I}$-LDL were between 500 and $2,000 \mathrm{cpm}$. They were handled in the same way as ${ }^{131}$ I-IDL. The majority of ${ }^{131}$ I-IDL points after $48 \mathrm{~h}$ and the majority of ${ }^{131} \mathrm{I}-\mathrm{LDL}$ points after $10 \mathrm{~d}$ were excluded. The specific activity of ${ }^{125} \mathrm{I}$ in LDL was measured in 18 samples of fasting plasma taken over the $14 \mathrm{~d}$ after the labeled LDL injection. All plasma samples taken after the return to the solid-food diet were left to stand overnight at $4^{\circ} \mathrm{C}$, and visible chylomicrons were removed with a pasteur pipette. Measurements of B48 contamination were not made. Six measurements of plasma insulin and unesterified fatty acid concentrations were made in the $5 \mathrm{~h}$ after the VLDL injections. 24-h urine collections were made to estimate LDL fractional clearance rate (FCR) from the ratio of radioactivity in urine and plasma. Plasma volume was calculated from the distribution volume of LDL calculated from plasma samples taken 10 , 20 , and $30 \mathrm{~min}$ after the LDL injection.

VLDL, IDL, and LDL were isolated by ultracentrifugation, and the specific activity of apoprotein B in each fraction was estimated after isopropanol extraction of apoprotein B and quantification by Lowry assay (19). Specific activity of VLDL triglyceride was measured in the pooled supernatants obtained during VLDL apoprotein B isolation. A first-order compartmental model was used to fit the specific activity/ time data using the CONSAM program (20). Rate constants for the VLDL apoB direct removal pathway $\left(K_{\mathrm{DR}}\right)$ and for the VLDL apoB to IDL apoB conversion pathway $\left(K_{\mathrm{VTIC}}\right)$ were calculated as the transport rate of each pathway divided by VLDL apoB pool size.

Statistics. All data are expressed as the mean \pm the standard error of the mean. The normality of distributions was tested by the Kolomo-
gorov-D statistic, and logorithms of non-normally distributed variables were used in analyses. The paired $t$ test was used to test the significance of differences between variables measured on the two diets. Pearsons product moment coefficient was used for correlations, except for variables with distributions not normalized by logarithmic transformation (the absolute values of fasting and 2-h glucose and insulin concentrations), when the Spearman rank order correlation coefficient was used. The residuals of regression analyses were normally distributed and there were no relationships between the residuals and either the predicted values or the independent variables. All statistical analyses were calculated using the Statistical Analysis System, SAS Institute Inc., Cary, NC.

\section{Results}

\section{Plasma concentrations of lipids, glucose, and insulin}

Fasting concentrations of plasma total lipids and lipoprotein lipids are presented in Table III. Plasma concentrations of total cholesterol and LDL cholesterol fell significantly on the high carbohydrate diet in both nondiabetic and diabetic groups. HDL cholesterol concentrations fell significantly on the highcarbohydrate diet in the nondiabetic group, but were unchanged in the diabetic group. Total and VLDL triglycerides were unchanged by the diet in both groups. The 24-hour triglyceride profiles were the same on both diets in both groups (Table III).

There were no changes in the means of fasting, 2-h, or 24-h glucose concentrations or of 24-h urinary glucose loss in either the nondiabetic or diabetic groups (Table IV). However, in four more severely diabetic subjects there were significant increases in the 24-h urinary glucose loss on the high-carbohydrate diet of between 29 and $158 \mathrm{~g}$ of glucose per day. Mean plasma insulin concentrations were the same on the two diets.

\section{Kinetics of apoB-containing lipoproteins}

$V L D L$ apoB and $V L D L$ triglycerides. In the nondiabetic subjects, the means for the concentration, production rate, and FCR of VLDL apoB and VLDL triglyceride were not significantly different on the two diets. However, the percentage of VLDL apoB that was directly removed from plasma $(49 \pm 2 \%$ vs. $62 \pm 2 \%, P<0.001)$ increased on the high-carbohydrate diet, and the percentage converted to IDL apoB decreased.

Table III. Changes in Mean Fasting Lipid and 24-h Triglyceride Concentrations

\begin{tabular}{lccc}
\hline & \multicolumn{2}{c}{ Nondiabetic } & Diabetic \\
\cline { 2 - 4 } & HIFAT & HICHO & HIFAT \\
\hline Total cholesterol $(m g / d l)$ & $186 \pm 9$ & $164 \pm 10^{\ddagger}$ & $185 \pm 17$ \\
Total triglyceride $(m g \cdot d / d l)$ & $135 \pm 10$ & $172 \pm 20$ & $178 \pm 50$ \\
24-h TG area $(m g \cdot d / d l)$ & $4,905 \pm 374$ & $5,482 \pm 686$ & $6,351 \pm 1,512$ \\
VLDL triglyceride $(m g \cdot d / d l)$ & $79 \pm 7$ & $106 \pm 12$ & $125 \pm 46$ \\
VLDL cholesterol $(m g / d l)$ & $19 \pm 1$ & $24 \pm 3$ & $23 \pm 6$ \\
VLDL phospholipid $(m g / d l)$ & $25 \pm 3$ & $32 \pm 4$ & $36 \pm 8$ \\
LDL cholesterol $(m g / d l)$ & $132 \pm 7$ & $111 \pm 8^{*}$ & $118 \pm 35$ \\
LDL triglyceride $(m g \cdot d / d l)$ & $49 \pm 6$ & $61 \pm 10$ & $25 \pm 5$ \\
HDL cholesterol $(m g / d l)$ & $35 \pm 1$ & $28 \pm 1^{*}$ & $130 \pm 15$ \\
& & $60 \pm 11$ & $32 \pm 3$
\end{tabular}

All values are given as mean \pm SEM. Abbreviations: HICHO, high-carbohydrate diet; HIFAT, high-fat diet. ${ }^{*} P<0.01 ;{ }^{\ddagger} P<0.001$. 
Table IV. Changes in Mean Glucose and Insulin Concentrations

\begin{tabular}{lcccc}
\hline & \multicolumn{2}{c}{ Nondiabetic } & \multicolumn{2}{c}{ Diabetic } \\
\cline { 2 - 5 } & HIFAT & HICHO & HIFAT & HICHO \\
\hline Fasting glucose $(m g / d l)$ & $95 \pm 4$ & $95 \pm 2$ & $214 \pm 33$ & $211 \pm 34$ \\
2-h glucose $(m g / d l)$ & $129 \pm 6$ & $139 \pm 12$ & $370 \pm 37$ & $348 \pm 43$ \\
24-h glucose area $(m g / d l)$ & $2,293 \pm 84$ & $2,260 \pm 52$ & $5,576 \pm 722$ & $5,733 \pm 871$ \\
24-h urine glucose $(m g / d l)$ & $0.4 \pm 0.1$ & $0.2 \pm 0.2$ & $55 \pm 23$ & $105 \pm 34$ \\
Fasting insulin $(\mu U / m l)$ & $55 \pm 14$ & $47 \pm 9$ & $42 \pm 14$ & $41 \pm 10$ \\
2-h insulin $(\mu U / m l)$ & $367 \pm 117$ & $237 \pm 32$ & $146 \pm 57$ & $158 \pm 61$ \\
\end{tabular}

All values are given as mean \pm SEM. Abbreviations: HICHO, high-carbohydrate diet; HIFAT, high-fat diet. ${ }^{*} P<0.01 ;{ }^{\ddagger} P<0.001$.

The rate constant $\left(K_{\mathrm{VTIC}}\right)$ for the VLDL apoB to IDL apoB conversion pathway decreased (Table $\mathrm{V}$ ).

In the diabetic group, the high-carbohydrate diet did not significantly change the mean concentration, production rate, or FCR of either VLDL apoB or VLDL triglyceride. However, as in the nondiabetic subjects, there was an increase in the mean percentage of VLDL apoB that was directly removed from plasma $(42 \pm 4 \%$ vs. $56 \pm 5 \%, P<0.001)$. There was a decrease in the mean conversion rate of VLDL apoB to IDL apoB and a decrease in the mean rate constant $\left(K_{\mathrm{VTIC}}\right)$ for this pathway (Table V).

In general the same trends in VLDL apoB metabolism occurred in both the nondiabetic and the diabetic subjects, which may justify combining the data for the two groups (Table V). When the data were combined there was a significant increase in the VLDL apoB pool size ( $303 \pm 20$ vs. $378 \pm 38$ $\mathrm{mg}$ VLDL apoB, $P=0.01$ ) on the high-carbohydrate diet. The increase in VLDL apoB concentrations did not reach statistical significance $(P=0.02)$. The difference between the change in pool size and the change in concentration was due to individual changes in the volume of distribution of VLDL apoB.
The mean production rate and the mean FCR for VLDL apoB were unchanged when the data were combined. The mean VLDL apoB to IDL apoB conversion rate and the mean rate constant for conversion of VLDL apoB to IDL apoB were decreased. The mean VLDL apoB direct uptake rate increased, and the rate constant for this pathway was unchanged. The change in VLDL apoB concentration was positively correlated with the change in the rate of direct uptake of VLDL apoB (Fig. 1).

There were no changes in either the mean VLDL triglyceride concentration or the mean VLDL triglyceride pool size on the high-carbohydrate diet when the groups were combined. The mean production rates and FCRs for VLDL triglyceride were also unchanged. The mean ratio of triglyceride to apoB in VLDL was unchanged, and the individual changes in this ratio did not correlate with any changes in VLDL metabolism.

Mean plasma insulin concentrations and mean plasma unesterified fatty acid concentrations were unchanged on the high-carbohydrate diet. Individual changes in insulin concentrations were correlated with individual changes in both the production rate $(r=0.74, P<0.01)$ and FCR $(r=0.84, P$

Table V. Mean VLDL Apoprotein B and Triglyceride Kinetic Data on High-Fat and High-Carbohydrate Diets

\begin{tabular}{|c|c|c|c|c|c|c|}
\hline & \multicolumn{2}{|c|}{ Nondiabetic } & \multicolumn{2}{|c|}{ Diabetic } & \multicolumn{2}{|c|}{ Combined } \\
\hline & HIFAT & HICHO & HIFAT & HICHO & HIFAT & HICHO \\
\hline \multicolumn{7}{|l|}{ Total VLDL } \\
\hline$[\mathrm{ApoB}](m g / d l)$ & $8.0 \pm 0.5$ & $9.5 \pm 1.1$ & $9.1 \pm 1.0$ & $10.7 \pm 2.0$ & $8.6 \pm 0.6$ & $10.1 \pm 1.1$ \\
\hline Flux (mg/kg ffm per day) & $16.1 \pm 1.5$ & $16.5 \pm 1.5$ & $22.2 \pm 1.5$ & $22.6 \pm 1.9$ & $19.2 \pm 1.5$ & $19.6 \pm 1.5$ \\
\hline FCR (pools per day) & $4.2 \pm 0.4$ & $3.6 \pm 0.4$ & $4.4 \pm 0.4$ & $4.3 \pm 0.6$ & $4.3 \pm 0.3$ & $4.0 \pm 0.3$ \\
\hline \multicolumn{7}{|l|}{ Direct removal } \\
\hline Flux (mg/kg ffm per day) & $7.8 \pm 0.8$ & $9.8 \pm 0.6$ & $9.2 \pm 1.0$ & $12.8 \pm 1.8$ & $8.5 \pm 1.0$ & $11.3 \pm 1.5^{*}$ \\
\hline$K_{\mathrm{DR}}$ & $2.1 \pm 0.2$ & $2.2 \pm 0.3$ & $1.9 \pm 0.3$ & $2.4 \pm 0.4$ & $2.0 \pm 0.3$ & $2.3 \pm 0.3$ \\
\hline \multicolumn{7}{|l|}{ VLDL to IDL conversion } \\
\hline Flux (mg/kg ffm per day) & $8.3 \pm 0.9$ & $6.6 \pm 0.9$ & $13.0 \pm 1.6$ & $9.8 \pm 1.1^{*}$ & $10.7 \pm 1.1$ & $8.3 \pm 0.9^{\ddagger}$ \\
\hline$K_{\mathrm{VTIC}}$ & $2.1 \pm 0.2$ & $1.3 \pm 0.2^{*}$ & $2.5 \pm 0.3$ & $1.9 \pm 0.3^{*}$ & $2.3 \pm 0.2$ & $1.6 \pm 0.2^{\S}$ \\
\hline \multicolumn{7}{|l|}{ VLDL triglyceride } \\
\hline [VLDL TG] $(m g / d l)$ & $127 \pm 10$ & $182 \pm 27$ & $167 \pm 35$ & $196 \pm 51$ & $147 \pm 18$ & $189 \pm 28$ \\
\hline Flux (mg/kg ffm per day) & $403 \pm 66$ & $496 \pm 116$ & $890 \pm 236$ & $744 \pm 168$ & $647 \pm 136$ & $620 \pm 104$ \\
\hline FCR (pools per day) & $6.5 \pm 0.9$ & $5.2 \pm 0.4$ & $8.9 \pm 1.3$ & $7.8 \pm 1.4$ & $7.7 \pm 0.8$ & $6.6 \pm 0.8$ \\
\hline
\end{tabular}

All values are given as mean \pm SEM. Abbreviations: HICHO, high-carbohydrate diet; HIFAT, high-fat diet. $K_{\mathrm{DR}}$ and $K_{\mathrm{VTIC}}$ are rate constants for the VLDL direct removal and VLDL to IDL conversion pathways (see Methods). ${ }^{*} P<0.01 ;{ }^{\ddagger} P<0.001 ;{ }^{8} P<0.0001$. 


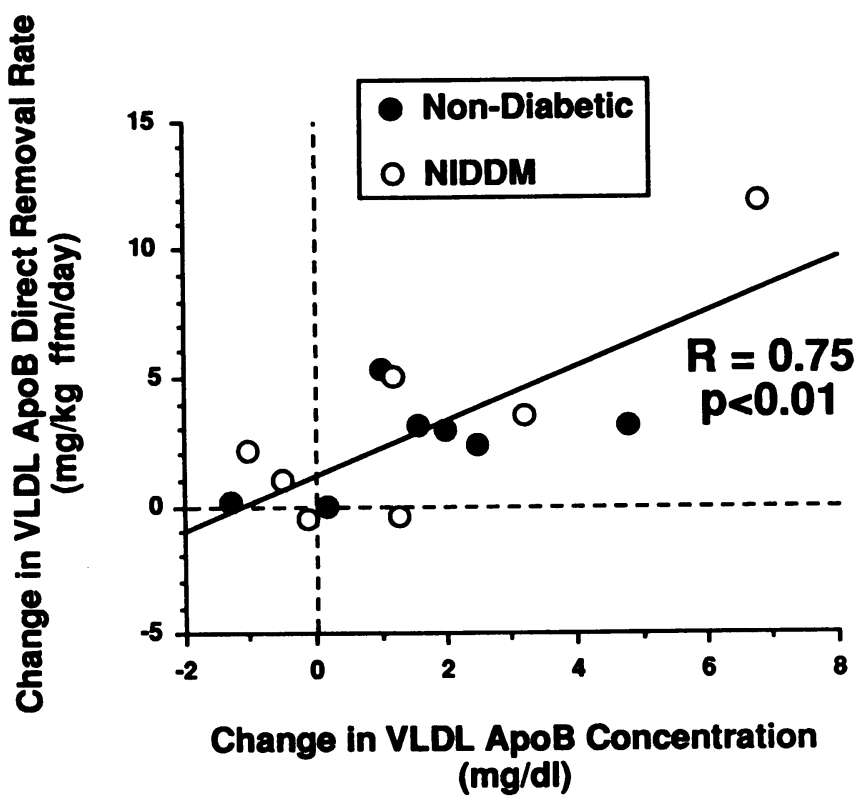

Figure 1. Individual changes in the direct removal rate of VLDL apo $B$ as a function of the changes in VLDL apoB concentration; $r$ $=0.75, P<0.01$, slope $=1.1 \pm 0.3$, intercept $=1.2 \pm 0.7$, NS.

$<0.001)$ of VLDL triglyceride. There were no correlations between individual changes in unesterified fatty acid concentrations and individual changes in VLDL metabolism.

$I D L a p o B$. Because of the decreased conversion rate of VLDL apoB to IDL apoB on the high-carbohydrate diet, the transport rate for IDL apoB was significantly decreased (Table VI). Even so, IDL apoB concentrations were not decreased because the FCR for IDL apoB tended to decrease on the high-carbohydrate diet. All of the label that passed from VLDL to LDL passed through IDL, and at least $95 \%$ of IDL apoB flux was converted to LDL apoB on both diets.

$L D L a p o B$. In the nondiabetic group (Table VII), there were significant decreases in mean LDL apoB and mean LDL cholesterol concentrations. There was a decrease in the mean production rate of LDL apoB from IDL, although the mean for total LDL production rate was unchanged, due to an increase in direct input of LDL in two subjects (1 and 4). There were no changes in either the mean $L D L$ apoB fractional clearance rate or the mean LDL cholesterol to apoB ratio.

In the diabetic group (Table VIII), there was a significant decrease in the mean LDL cholesterol concentration, and LDL apoB concentrations tended to decrease. As in the nondiabetic group, there was a decrease in the mean production rate of LDL apoB from IDL, although the mean for total LDL production rate was unchanged, due to an increase in direct input of LDL in two subjects ( 9 and 11). There were no changes in either the mean LDL apoB fractional clearance rate or the mean LDL cholesterol to apoB ratio.

When the data were combined, LDL apoB concentrations were decreased $(P<0.01)$, but there were no changes in the total LDL apoB production rate $(P=0.06)$ or in the LDL apoB fractional clearance rate $(P=0.15)$. All subjects had a decrease in the conversion rate of VLDL apoB to LDL apoB on the high-carbohydrate diet. However, four subjects had an increase in direct input of LDL apoB that more than offset the decreased input from IDL. There was a correlation between the change in LDL apoB production rate and the change in LDL apoB concentration (Fig. 2), and this relationship had a significant negative $y$-intercept.

\section{Discussion}

There are three noteworthy findings in this study. First, the high-carbohydrate diet induced a decrease in LDL cholesterol concentrations in all subjects, and this decrease was associated with an increase in the VLDL apoB pool size and with a decrease in the flux and the rate constant $\left(K_{\mathrm{VTIC}}\right)$ of the pathway that converts VLDL into IDL and LDL. Secondly, we could find no evidence that a solid-food, isocaloric, high-carbohydrate, low-saturated-fat diet causes an increase in VLDL production by the liver. Thirdly, the metabolic responses of the diabetic and nondiabetic subjects to the high carbohydrate diet were very similar.

Mechanisms for the decrease in LDL cholesterol concentrations. The study clearly identified one consistent set of metabolic changes that contributed to the fall in LDL cholesterol concentrations in all subjects. The conversion rate of VLDL apoB to IDL apoB and LDL apoB was decreased in all subjects. The rate constant for the VLDL to IDL conversion pathway $\left(K_{\mathrm{VTIC}}\right)$ was also significantly decreased. A simultaneous decrease in the flux and the rate constant for this pathway strongly suggests that one or more of the mechanisms for converting VLDL to IDL was impaired by the high-carbohydrate diet. The increase in VLDL apoB pool size is a logical consequence of a decrease in VLDL metabolism in the presence of an unchanged VLDL production rate. An increase in VLDL apoB with a high-carbohydrate diet has also been found by other investigators (21-24). Since the conversion of VLDL to IDL was decreased, there was a significant increase in the rate of direct uptake of VLDL by the liver, which is the major alternative pathway for VLDL metabolism. This finding has been previously reported in hypertriglyceridemic subjects by

Table VI. Mean IDL Apoprotein B Kinetic Data on High-Fat and High Carbohydrate Diets

\begin{tabular}{|c|c|c|c|c|c|c|}
\hline & \multicolumn{2}{|c|}{ Nondiabetic } & \multicolumn{2}{|c|}{ Diabetic } & \multicolumn{2}{|c|}{ Combined } \\
\hline & HIFAT & HICHO & HIFAT & HICHO & HIFAT & HICHO \\
\hline$[\mathrm{ApoB}](m g / d l)$ & $3.2 \pm 0.3$ & $3.7 \pm 0.4$ & $3.8 \pm 0.5$ & $3.8 \pm 0.6$ & $3.5 \pm 0.3$ & $3.8 \pm 0.3$ \\
\hline Flux ( $m g / k g f f m$ per day) & $8.3 \pm 0.9$ & $6.6 \pm 0.9$ & $13.0 \pm 1.6$ & $10.1 \pm 1.2^{*}$ & $10.7 \pm 1.1$ & $8.4 \pm 0.9^{\ddagger}$ \\
\hline FCR (pools per day) & $5.6 \pm 0.7$ & $3.9 \pm 0.7^{*}$ & $6.1 \pm 0.9$ & $5.5 \pm 1.9$ & $5.9 \pm 0.6$ & $4.7 \pm 0.6$ \\
\hline
\end{tabular}

All values are given as mean \pm SEM. Abbreviations: HICHO, high-carbohydrate diet; HIFAT, high-fat diet. ${ }^{*} P<0.01 ;{ }^{\ddagger} P<0.001 ;{ }^{\S} P<0.0001$. 
Table-VII. Changes in LDL Concentrations, Composition, and Kinetics in Nondiabetic Subjects

\begin{tabular}{|c|c|c|c|c|c|c|c|c|c|}
\hline \multirow[b]{2}{*}{ Subject } & \multirow[b]{2}{*}{ Diet } & \multirow[b]{2}{*}{ [ApoB] } & \multirow[b]{2}{*}{ [Chol] } & & \multirow[b]{2}{*}{$\begin{array}{l}\text { Flux from } \\
\text { IDL }\end{array}$} & \multirow[b]{2}{*}{ FCR } & \multicolumn{2}{|c|}{ Direct synthesis } & \multirow[b]{2}{*}{$\begin{array}{c}\text { Cholesterol } \\
\text { to apoB ratio }\end{array}$} \\
\hline & & & & Total flux & & & Flux : & $\%$ total & \\
\hline & : & \multicolumn{2}{|c|}{$m g / d l$} & \multicolumn{2}{|c|}{$m g / k g$ ffm per day } & $\begin{array}{c}\text { pools per } \\
\text { day }\end{array}$ & $\begin{array}{c}\text { mg/kg } \\
\text { ffm per } \\
\text { day }\end{array}$ & & \\
\hline \multirow[t]{2}{*}{1} & HIFAT & 70 & 133 & 9.8 & 9.2 & 0.30 & 0.6 & 6 & 1.89 \\
\hline & HICHO & 74 & 111 & 11.6 & 8.7 & 0.31 & 2.9 & 25 & 1.49 \\
\hline \multirow[t]{2}{*}{2} & HIFAT & 54 & 69 & 12.9 & 6.8 & 0.43 & 6.1 & 47 & 1.27 \\
\hline & HICHO & 44 & 57 & 8.2 & 3.4 & 0.33 & 4.8 & 58 & 1.30 \\
\hline \multirow[t]{2}{*}{3} & HIFAT & 78 & 123 & 12.0 & 6.7 & 0.30 & 5.3 & 44 & 1.59 \\
\hline & НICHO & 58 & 106 & 8.2 & 4.3 & 0.28 & 3.9 & 47 & 1.85 \\
\hline \multirow[t]{2}{*}{4} & HIFAT & 87 & 119 & 16.9 & 12.5 & 0.41 & 4.4 & 26 & 1.37 \\
\hline & НICHO & 77 & 91 & 17.9 & 8.9 & 0.48 & 9.0 & 50 & 1.18 \\
\hline \multirow[t]{2}{*}{5} & HIFAT & 74 & 82 & 15.6 & 9.0 & 0.39 & 6.6 & 42 & 1.11 \\
\hline & HICHO & 58 & 69 & 13.6 & 8.2 & 0.43 & 5.4 & 40 & 1.19 \\
\hline \multirow[t]{2}{*}{6} & HIFAT & 60 & 87 & 9.1 & 5.8 & 0.32 & 3.3 & 36 & 1.45 \\
\hline & HICHO & 46 & 83 & 7.6 & 4.4 & 0.36 & 3.2 & 42 & 1.82 \\
\hline 7 & HIFAT & 62 & 108 & 10.0 & 8.0 & 0.26 & 2.0 & 20 & 1.72 \\
\hline & HICHO & 52 & 90 & 8.9 & 7.3 & 0.29 & 1.6 & 18 & 1.75 \\
\hline \multirow[t]{2}{*}{ Mean \pm SEM } & HIFAT & $69 \pm 4$ & $103 \pm 9$ & $12.3 \pm 1.1$ & $8.3 \pm 0.8$ & $0.34 \pm 0.02$ & $4.0 \pm 0.8$ & $32 \pm 6$ & $1.49 \pm 0.10$ \\
\hline & HICHO & $58 \pm 5^{*}$ & $87 \pm 7^{*}$ & $10.9 \pm 1.4$ & $6.5 \pm 0.9^{\ddagger}$ & $0.35 \pm 0.02$ & $4.4 \pm 0.9$ & $40 \pm 5$ & $1.51 \pm 0.11$ \\
\hline
\end{tabular}

Abbreviations: Chol, cholesterol; HICHO, high-carbohydrate diet; HIFAT, high-fat diet. ${ }^{*} P<0.01 ;{ }^{\ddagger} P<0.001 ;{ }^{\S} P<0.0001$.

Ginsberg et al. (21). The individual increases in the rate of VLDL apoB direct removal were positively correlated with the individual increases in VLDL apoB concentration (Fig. 1). This positive correlation suggests that the increase in VLDL apoB direct removal was a consequence of the increase in VLDL apoB concentrations.
It might be argued that the high-carbohydrate diet decreased the conversion rate of VLDL to IDL by increasing the activity of the VLDL direct uptake pathway, leaving less VLDL to be converted to IDL and LDL. If this were the case, then a decrease in VLDL apoB pool size would have been expected, and the correlation between the change in VLDL

Table VIII. Changes in LDL Concentrations, Composition, and Kinetics in Diabetic Subjects

\begin{tabular}{|c|c|c|c|c|c|c|c|c|c|}
\hline \multirow[b]{2}{*}{ Subject } & \multirow[b]{2}{*}{ Diet } & \multirow[b]{2}{*}{ [ApoB] } & \multirow{2}{*}{ [Chol] } & \multirow[b]{2}{*}{ Total flux } & \multirow{2}{*}{$\begin{array}{l}\text { Flux from } \\
\text { IDL }\end{array}$} & \multirow[b]{2}{*}{ FCR } & \multicolumn{2}{|c|}{ Direct synthesis } & \multirow{2}{*}{$\begin{array}{c}\text { Cholesterol } \\
\text { to apoB } \\
\text { ratio }\end{array}$} \\
\hline & & & & & & & Flux & $\%$ total & \\
\hline & & & & $m g / k$ & ser day & $\begin{array}{c}\text { pools per } \\
\text { day }\end{array}$ & $\begin{array}{c}\mathrm{mg} / \mathrm{kg} \\
\text { fm per } \\
\text { day }\end{array}$ & & \\
\hline \multirow[t]{2}{*}{8} & HIFAT & 66 & 102 & 11.8 & 7.4 & 0.44 & 4.8 & 41 & 1.56 \\
\hline & HICHO & 53 & 84 & 10.4 & 6.0 & 0.45 & 4.4 & 42 . & 1.59 \\
\hline \multirow[t]{2}{*}{9} & HIFAT & 45 & 61 & 14.9 & 10.6 & 0.63 & 4.3 & 29 & 1.37 \\
\hline & HICHO & 47 & 53 & 17.0 & 6.3 & 0.74 & 10.7 & 63 & 1.12 \\
\hline \multirow[t]{2}{*}{10} & HIFAT & 79 & 126 & 14.1 & 11.6 & 0.30 & 2.5 & 18. & 1.59 \\
\hline & HICHO & 76 & 112 & 13.6 & 9.8 & 0.31 & 3.8 & 28 & 1.47 \\
\hline \multirow[t]{2}{*}{11} & HIFAT & 61 & 74 & 16.6 & 16.6 & 0.37 & 0.0 & 0 & 1.20 \\
\hline & HICHO & 60 & 65 & 19.7 & 11.6 & 0.46 & 8.1 & 41 & 1.09 \\
\hline \multirow[t]{2}{*}{12} & HIFAT & 57 & 102 & 15.0 & 9.6 & 0.43 & 5.4 & 36 & 1.79 \\
\hline & HICHO & 44 & 75 & 10.6 & 9.3 & 0.41 & 1.3 & 12 & 1.69 \\
\hline \multirow[t]{2}{*}{13} & HIFAT & 73 & 100 & 14.6 & 11.5 & 0.36 & 3.1 & 21 & 1.37 \\
\hline & HICHO & 58 & 77 & 13.4 & 9.9 & 0.42 & 3.5 & 26 & 1.33 \\
\hline \multirow[t]{2}{*}{14} & HIFAT & 113 & 188 & 18.9 & 17.6 & 0.29 & 1.3 & 7 & 1.67 \\
\hline & HICHO & 110 & 177 & 17.4 & 14.4 & 0.27 & 3.0 & 17 & 1.61 \\
\hline \multirow[t]{2}{*}{ Mean \pm SEM } & HIFAT & $70 \pm 8$ & $108 \pm 16$ & $15.1 \pm 0.8$ & $12.1 \pm 1.4$ & $0.40 \pm 0.04$ & $3.1 \pm 0.7$ & $22 \pm 6$ & $1.51 \pm 0.08$ \\
\hline & HICHO & $64 \pm 9$ & $92 \pm 16^{*}$ & $14.6 \pm 1.3$ & $9.6 \pm 1.1^{\ddagger}$ & $0.44 \pm 0.06$ & $5.0 \pm 1.2$ & $33 \pm 7$ & $1.41 \pm 0.09$ \\
\hline
\end{tabular}

Abbreviations: Chol, cholesterol; HICHO, high-carbohydrate diet; HIFAT, high-fat diet. ${ }^{*} P<0.01 ;{ }^{\ddagger} P<0.001 ;{ }^{\S} P<0.0001$. 


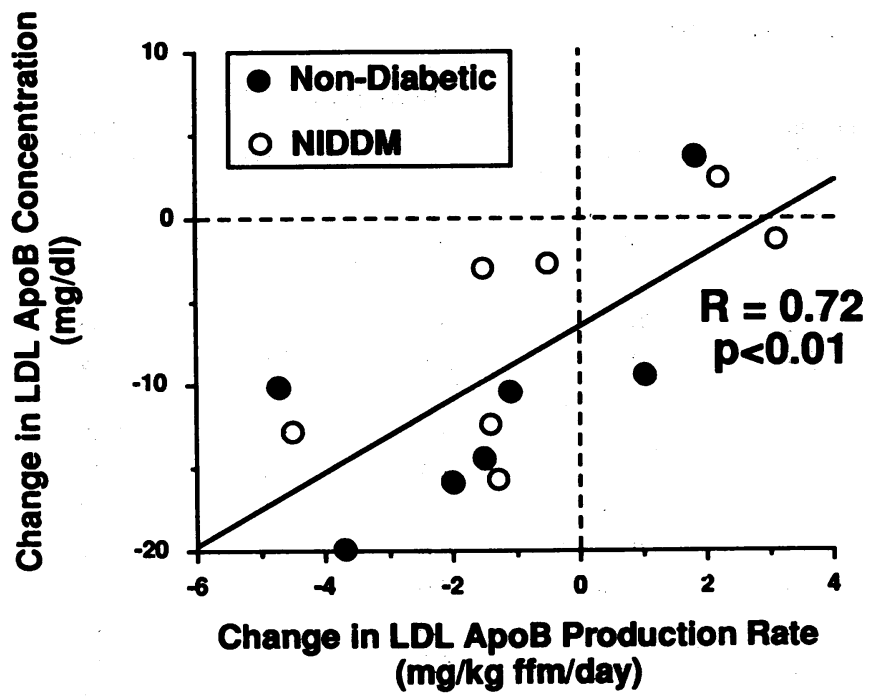

Figure 2. Individual changes in $\mathrm{LDL}$ apoB concentration as a function of changes in $\mathrm{LDL}$ apoB production rate; $r=0.72, P<0.01$, slope $=0.03 \pm 0.01$, intercept $=-5.9 \pm 1.6, \mathrm{p}<0.01$.

apoB concentration size and the change in VLDL direct uptake should have been negative. Other findings suggesting that the activity of the VLDL direct uptake pathway was unchanged by the high-carbohydrate diet are the unchanged rate constant $\left(K_{\mathrm{DU}}\right)$ for this pathway, and the observation that the $y$-intercept for the regression between the changes in VLDL pool size and VLDL direct uptake (Fig. 1) could not be distinguished from zero; i.e., we could not detect any change in VLDL direct uptake that was independent of the change in VLDL apoB concentrations.

We do not know the exact mechanism by which the highcarbohydrate diet impaired the conversion of VLDL to IDL but the mechanism must involve removal of triglyceride from VLDL particles. Possibilities include a change in VLDL composition that decreased the affinity of VLDL for lipoprotein lipase or hepatic lipase, a decrease in activity of either hepatic lipase or of lipoprotein lipase (25), or a decrease in the exchange of VLDL triglyceride for HDL cholesterol. We were unable to find any changes in VLDL composition in terms of the ratios of cholesterol, triglyceride, phospholipid and apoprotein B (data not shown). Other investigators have reported changes in the apolipoprotein composition of plasma on a high-carbohydrate diet $(22,26)$ that may influence lipolysis. A decrease in the activity of lipoprotein lipase in postheparin plasma has also been reported $(27,28)$.

The VLDL triglyceride data are consistent with the hypothesis that the high-carbohydrate diet decreased the activity of mechanisms that remove triglyceride from VLDL, even though the mean FCR for VLDL triglyceride did not decrease. The FCR for VLDL triglyceride is influenced by both the activity and the degree of saturation of all processes that remove VLDL triglyceride from plasma. These processes include removal of whole VLDL particles from the plasma, the triglyceride content of the particles being preferentially removed, lipolysis of triglyceride, and cholesterol/triglyceride exchange with HDL. A decrease in triglyceride removal from VLDL would tend to decrease the mean FCR for VLDL triglyceride. However, this decrease could have been offset by either of two factors. First, there could be an increase in the triglyceride content of the VLDL particles being preferentially removed by the hepatic direct uptake pathway. This is supported by the finding that although mean VLDL apoB pool size increased on the high-carbohydrate diet, there was no change in the VLDL triglyceride pool size. Secondly, the changes in activity of the VLDL direct removal pathway $\left(K_{\mathrm{DU}}\right)$ were very variable in this study. Two subjects had particularly large increases in $K_{\mathrm{DU}}$ associated with increases in VLDL triglyceride FCR (individual data not shown). Both of these individuals had a decrease in the flux and the rate constant for VLDL conversion of apoB to IDL apoB, which may be a better index of a change in activity of mechanisms that remove triglyceride from VLDL.

Although a decrease in conversion of VLDL to IDL was a consistent finding in all subjects studied, it probably does not account for all of the decrease in LDL cholesterol concentrations. There is evidence for two other mechanisms. Fig. 2 suggests that there was an increase in activity of LDL apoB clearance mechanisms on the high-carbohydrate diet. There was a significant negative $y$-intercept in the regression between the change in $\mathrm{LDL}$ apoB production rate and the change in LDL apoB concentration. In those subjects who had no overall decrease in LDL production rate, there was still a decrease in LDL apoB pool size. This implies that there was an increase in activity of the mechanisms that clear LDL from plasma, at least in those subjects whose data points lie close to the $y$-axis. The possible causes of an increase in clearance are an increase in affinity of LDL particles for the LDL receptor, and an increase in LDL receptor number. A high-carbohydrate diet has been reported to increase affinity of LDL particles for the LDL receptor (29). ${ }^{2}$ This is probably the cause of the increase in activity of LDL clearance mechanisms that we found. An increase in LDL receptor number cannot be excluded, but seems less likely as there was no evidence in the VLDL and IDL data for an increase in the activity of mechanisms that directly remove VLDL and IDL from the plasma.

Four subjects had an increased direct input of $L D L$ apoB, with no overall change in LDL apoB concentration. The decrease in LDL cholesterol concentrations in these subjects was thus dependent on a decrease in the ratio of LDL cholesterol to apoB. This change was not noted as consistently in our study as it has been by previous investigators $(21,22,30)$, probably because of differences in the dietary modifications used.

High-carbohydrate diets and VLDL production. Increased plasma triglyceride concentrations are associated with atherosclerosis in patients with NIDDM $(11,12)$. Thus, with regard to the safety of high-carbohydrate diets, it is important to know that they do not increase either the concentration or the flux of potentially atherogenic VLDL particles. Although VLDL apoB pool size was increased in this study, it was not because of an increase in production rate of VLDL particles by the liver. It is not known whether this increase in VLDL apoB pool size is associated with an increased risk for atherosclerosis. It is also not known whether this increase in VLDL apoB pool size would occur on a high-carbohydrate diet with an $\mathrm{ad}$ libitum caloric intake.

2. Hara, H., W. G. H. Abbott, L. Patti, G. Ruotolo, B. A. Swinburn, Rose M. Fields, S. Kataoka, and B. V. Howard. Increased receptor binding of LDL from individuals consuming a high carbohydrate, low saturated fat diet. (Manuscript submitted for publication.) 
An increase in plasma and VLDL triglyceride concentrations with isocaloric high-carbohydrate diets has been reported in some but not all high carbohydrate diet studies (8). The mechanism for this carbohydrate-induced hypertriglyceridemia is not definitely known. Evidence for a decrease in VLDL triglyceride metabolism can usually be found in kinetic studies of subjects on high-carbohydrate diets (31-34). Our data also indicate that a decrease in metabolism of VLDL occurs. The failure to find an increase in VLDL triglyceride concentrations suggests that the increase in VLDL removal by the liver was sufficient to counteract the decrease in metabolism. An increase in VLDL triglyceride production rate has usually been found in kinetic studies of subjects who develop carbohydrate-induced hypertriglyceridemia $(24,33,34)$, and an increase in VLDL triglyceride production rate is probably necessary for carbohydrate-induced hypertriglyceridemia to occur. Increased VLDL production rates have been noted with sucrose compared with starch (31), and with saturated fats compared with unsaturated fats (35). Isocaloric high-carbohydrate diet studies that increase simple sugars $(24,33,34,36)$ or reduce unsaturated fatty acids $(36,37)$ may be more likely to show a carbohydrate-induced rise in triglyceride concentrations.

In summary, there were no significant differences between the diabetic and the nondiabetic subjects in the response of apoB and triglyceride kinetics to a high-carbohydrate, low-saturated-fat diet. This diet did not induce hypertriglyceridemia, and there was no increase in the production rate of VLDL apoB or VLDL triglyceride. Three mechanisms could be identified that contributed to the decrease in LDL cholesterol concentrations. A decrease in the activity of the mechanisms that convert VLDL to IDL and LDL was a significant factor in all subjects. This could be due to a decrease in lipolysis of VLDL triglyceride, or to a decrease in triglyceride exchange with HDL. An increase in the activity of mechanisms that clear LDL apoB from plasma, and a decrease in the LDL cholesterol to apoB ratio also contributed to the decrease in LDL cholesterol concentrations in some subjects.

\section{Acknowledgments}

We wish to thank Ms. Carol Lamkin, Ms. Vicky Boyce, and the nursing and dietary staff of the Clinical Diabetes and Nutrition Section, Phoenix; Thomas Anderson, John Brown, Rose Marie Fields, Kathy Steffen, Victoria Ossowski, Vera Rodriguez, and Deborah WolfeLopez for technical assistance; and the Gila River Indian Community. CONSAM computer programs were kindly provided by the U.S. Public Health Service/National Institutes of Health/National Heart, Lung and Blood Institute-National Cancer Institute development project.

This study was supported in part by a grant from the Arizona Disease Control Commission.

\section{References}

1. Grundy, S. M., N. Dana, M. F. Whelan, and L. Franklin. 1986. Comparison of three cholesterol-lowering diets in normolipidemic men. JAMA (J. Am. Med. Assoc.) 256:2351-2355.

2. Cortese, C., Y. Levy, E. D. Janus, P. R. Turner, S. N. Rao, N. E. Miller, and B. Lewis. 1983. Modes of action of lipid-lowering diets in man: studies of apolipoprotein B kinetics in relation to fat consumption and dietary fatty acid composition. Eur. J. Clin. Invest. 13:79-85.
3. Shepherd, J., C. J. Packard, S. M. Grundy, D. Yeshurun, A. M. Gotto, Jr., and O. D. Taunton. 1980. Effects of saturated and polyunsaturated fat diets on the chemical composition and metabolism of low density lipoproteins in man. J. Lipid Res. 21:91-99.

4. Turner, J. D., Ngoc-Anh Le, and V. W. Brown. 1981. Effect of changing dietary fat saturation on low density lipoprotein metabolism in man. Am. J. Physiol. 241:E57-E63.

5. Spady, D. K., and J. M. Dietschy. 1988. Interaction of dietary cholesterol and triglycerides in the regulation of hepatic low density lipoprotein transport in the hamster. J. Clin. Invest. 81:300-309.

6. Grundy, S. M. 1987. Monounsaturated fatty acids, plasma cholesterol, and coronary heart disease. Am. J. Clin. Nutr. 45:1168-1175.

7. Brown, W. V., and W. Karmally. 1985. Coronary heart disease and the consumption of diets high in wheat and other grains. Am: J. Clin. Nutr. 41:1163-1171.

8. Abbott, W. G. H., V. L. Boyce, S. M. Grundy, and B. V. Howard. 1989. Effects of replacing saturated fat with complex carbohydrate in diets of subjects with NIDDM. Diabetes Care. 12:102-107.

9. Steering Committee of the American Heart Association. 1986. Dietary guidelines for healthy American adults. Circulation. 74:1465A-1468A.

10. American Diabetes Association. 1987. Nutritional recommendations and principles for individuals with diabetes mellitus. Diabetes Care. 10:126-132.

11. West, K. M., M. M. S. Ahuja, P. H. Bennett, A. Czyzyk, O. Mateo de Acosta, J. H. Fuller, B. Grab, V. Grabauskas, J. Jarrett, K. Kosaka, et al. 1983. The role of circulating glucose and triglyceride concentrations and their interaction with other risk factors as determinants of arterial disease in nine diabetic population samples form the WHO multinational study. Diabetes Care. 6:361-369.

12. Fontbonne, A. E. Eschwege, F. Cambien, J.-L. Richard, P. Ducimetiere, N. Thibult, J.-M. Warnet, J.-R. Claude, and G.-E. Rosselin. 1989. Hypertriglyceridemia as a risk factor of coronary heart disease mortality in subjects with impaired glucose tolerance or diabetes: results from the 11-year follow-up of the Paris prospective study. Diabetologia. 32:300-304.

13. WHO Expert Committee. 1980. Diabetes Mellitus Second Report. World Health Organization, Geneva. WHO Tech. Rep. Ser. 646

14. Pennington, J., and H. N. Church. 1985 Bowes and Church's Food Values of Portions Commonly Used. 14th edition. J. P. Lippincott Co., Philadelphia. 1985.

15. U. S. Dept. of Agriculture. 1980. Composition of Foods. USDA Agriculture Handbook No. 8-1 through 8-9. USDA, Washington, DC.

16. Abbott, W. G. H., S. Lillioja, A. A. Young, J. K. Zawadski, H Yki-Jarvinen, L. Christin, and B. V. Howard. 1987. Relationships between plasma lipoprotein concentrations and insulin action in an obese hyperinsulinemic population. Diabetes. 36:897-904.

17. Egusa, G., W. F. Beltz, S. M. Grundy, and B. V. Howard. 1985 Influence of obesity on the metabolism of apolipoprotein $B$ in human.s J. Clin. Invest. 76:596-603.

18. McFarlane, A. S. 1958. Efficient trace-labelling of proteins with iodine. Nature (Lond.) 182:53.

19. Egusa, G., D. W. Brady, S. M. Grundy, and B. V. Howard. 1983. Isopropanol precipitation method for the determination of apolipoprotein B specific activity and plasma concentrations during metabolic studies of very low density lipoprotein and low density lipoprotein apolipoprotein B. J. Lipid Res. 24:1261-1267.

20. Beltz, W. F., Y. A. Kesaniemi, B. V. Howard, and S. M. Grundy. 1985. Development of an integrated model for analyses of the kinetics of apolipoprotein B in plasma very low density lipoproteins, intermediate density lipoproteins and low density lipoproteins. J. Clin. Invest. 76:575-585.

21. Ginsberg, H. N., Ngoc-Anh Le, J. Melish, D. Steinberg, and W. V. Brown. 1981. Effect of a high carbohydrate diet on apoprotein B catabolism in man. Metab. Clin. Exp. 30:347-353.

22. Schonfeld, G., S. W. Weidman, J. L. Witzum, and R. M. Bowen. 1976. Alterations in levels and interrelations of plasma apolipoproteins induced by diet. Metab. Clin. Exp. 25:261-275. 
23. Huff, M. W., and P. J. Nestel. 1982. Metabolism of apolipoproteins $\mathrm{CII}, \mathrm{CIII}_{1}, \mathrm{CIII}_{2}$, and VLDL-B in human subjects consuming high carbohydrate diets. Metab. Clin. Exp. 31:493-498.

24. Melish, J., Ngoc-Anh Le, H. N. Ginsberg, D. Steinberg, and W. V. Brown. 1980. Dissociation of apoprotein B and triglyceride production in very low density lipoproteins. Am. J. Physiol. E354E362.

25. Goldberg, I. J., Ngoc-Anh Le, H. N. Ginsberg, R. M. Krauss, and F. T. Lindgren. 1988. Lipoprotein metabolism during acute inhibition of lipoprotein lipase in the cynomolgus monkey. J. Clin. Invest. 81:561-568.

26. Kashyap, M. L., R. L. Barnhart, L. S. Srivastava, G. Perisutti, P. Vink, C. Allen, E. Hogg, D. Brady, C. J. Glueck, and R. L. Jackson. 1982. Effects of dietary carbohydrates and fat on plasma lipoproteins and apolipoproteins C-II and C-III in healthy men. J. Lipid Res. 23:877-886.

27. Fredrickson, D. S., K. Ono, and L. L. Davis. 1963. Lipolytic activity of post-heparin plasma in hyperglyceridemia. J. Lipid Res. 4:24-33.

28. Jackson, R. L., M. T. Yates, C. A. McNerney, and M. L. Kashyap. 1987. Diet and HDL metabolism: high carbohydrate vs. high fat diets. Adv. Exp. Med. Biol. 210:165-172.

29. Keidar, S., A. C. Goldberg, K. Cook, J. Bateman, and G. Schonfeld. 1989. High carbohydrate fat-free diet modulates epitope expression of LDL-apoB-100 and interaction of LDL with human fibroblasts. J. Lipid Res. 30:1331-1339.
30. Wilson, D. E., and R. S. Lees. 1972. Reciprocal changes in the concentrations of very low and low density lipoproteins in man. $J$. Clin. Invest. 51:1051-1057.

31. Nestel, P. J., K. F. Carroll, and N. Havenstein. 1970. Plasma triglyceride response to carbohydrates, fats and caloric intake. Metab. Clin. Exp. 19:1-18.

32. Nestel, P. J. 1973. Triglyceride turnover in man. Prog. Biochem. Pharmacol. 8:125-160.

33. Reaven, G. M., D. B. Hill, R. C. Gross, and J. W. Farquhar. 1965. Kinetics of triglyceride turnover of very low density lipoproteins of human plasma. J. Clin. Invest. 44:1826-1833.

34. Quarfordt, S. H., A. Frank, D. M. Shames, M. Berman, and D. Steinberg. 1970. Very low density lipoprotein triglyceride transport in type IV hyperlipoproteinemia and the effects of carbohydrate rich diets. J. Clin. Invest. 49:2281-2297.

35. Chait, A., A. Onitiri, A. Nicoll, E. Rabaya, J. Davies, and B. Lewis. 1974. Reduction of serum triglyceride levels by polyunsaturated fat. Atherosclerosis. 20:347-364.

36. Coulston, A. M., C. B. Hollenbeck, A. L. M. Swislocki, Y.-D. I. Chen, and G. M. Reaven. 1987. Deleterious metabolic effects of highcarbohydrate, sucrose-containing diets in patients with non-insulindependent diabetes meliitus. Am. J. Med. 82:213-220.

37. Garg, A., A. Bonanome, S. M. Grundy, Z.-J. Zhang, and R. H. Unger. 1988. Comparison of a high-carbohydrate diet with a highmonounsaturated-fat diet in patients with non-insulin dependent diabetes mellitus. N. Engl. J. Med. 319:829-834. 